\title{
Statistical Assessment of the Impact of Renewable Energy Sources on Transient Stability
}

DOI:

10.1109/ISGTEurope.2018.8571762

\section{Document Version}

Accepted author manuscript

Link to publication record in Manchester Research Explorer

\section{Citation for published version (APA):}

Morales Alvarado, J., Milanovic, J. V., \& Papadopoulos, P. (2018). Statistical Assessment of the Impact of Renewable Energy Sources on Transient Stability. In 2018 IEEE PES Innovative Smart Grid Technologies Conference Europe (ISGT-Europe) https://doi.org/10.1109/ISGTEurope.2018.8571762

\section{Published in:}

2018 IEEE PES Innovative Smart Grid Technologies Conference Europe (ISGT-Europe)

\section{Citing this paper}

Please note that where the full-text provided on Manchester Research Explorer is the Author Accepted Manuscript or Proof version this may differ from the final Published version. If citing, it is advised that you check and use the publisher's definitive version.

\section{General rights}

Copyright and moral rights for the publications made accessible in the Research Explorer are retained by the authors and/or other copyright owners and it is a condition of accessing publications that users recognise and abide by the legal requirements associated with these rights.

\section{Takedown policy}

If you believe that this document breaches copyright please refer to the University of Manchester's Takedown Procedures [http://man.ac.uk/04Y6Bo] or contact uml.scholarlycommunications@manchester.ac.uk providing relevant details, so we can investigate your claim.

\section{OPEN ACCESS}




\section{Statistical Assessment of the Impact of Renewable Energy Sources on Transient Stability}

\author{
Juan D. Morales, Student Member, IEEE \\ Jovica V. Milanović, Fellow, IEEE \\ School of Electrical and Electronic Engineering \\ The University of Manchester \\ Manchester, UK \\ juan.morales@manchester.ac.uk \\ jovica.milanovic@manchester.ac.uk
}

\author{
Panagiotis N. Papadopoulos, Member, IEEE \\ Department of Electronic and Electrical Engineering \\ University of Strathclyde \\ Glasgow, UK \\ panagiotis.papadopoulos@strath.ac.uk
}

\begin{abstract}
This paper studies the impact of penetration of Renewable Energy Sources (RES) on the dynamic behavior of large power systems. Statistical analysis of the number of instabilities and the time of occurrence of loss of synchronism in the network was performed following large number of Monte Carlo simulations and considering high degrees of uncertainty. A criterion for instability detection is introduced. Conclusions are drawn with respect to the influence of RES on transient stability, the identification of critical generators and how these generators and their behavior may change for a wide range of RES penetration levels.
\end{abstract}

Index Terms-Corrective control, renewable energy sources, statistical transient stability assessment, time to instability

\section{INTRODUCTION}

Due to the increasing penetration of Renewable Energy Sources (RES) and displacing conventional generation in large power networks, research related to analyzing the impact of the inertia reduction in power systems becomes crucial. It is possible that in the close future the amount of conventional generation might not be enough to ensure the stable network operation after major disturbances.

Previous work on the impact of RES (mainly wind and solar technologies) on transient stability includes e.g., [1-5]. In these studies deterministic approaches are used for the assessment of specific mechanisms influencing the dynamic behavior of a power system. However, due to the high levels of uncertainty inherent to RES generation, a probabilistic approach seems to be the best option for a comprehensive analysis [6]. Monte Carlo (MC) based methods are used in [7, 8] to calculate the probability of instability, including wind uncertainties and in [9] to study the impact of RES, by analyzing certain transient stability indices and critical unstable generators. Non MC approaches [10-12] rely on analytical calculations of the probability of stability or stability margins, the use of energy functions and simplified Single Machine Equivalent (SIME) methods for stability assessment. However, these methods are usually less accurate compared to MC simulations and it is not easy to include detailed RES models with their associated controllers.

This paper uses time domain MC simulations to study system stability and focuses, for the first time, on the times of instability of synchronous generators as a statistical variable for analysis. The objective of the study is to obtain a more practical insight into the effects of increasing RES penetration that can be used for development of corrective control measures (e.g. the ones in [13]) to improve system stability. The identification of the time (following the fault in the system) at which each generator or groups of generators lose synchronism is an important step in that direction. All simulations are carried out in DigSilent/PowerFactory environment [14] using a modified version of the IEEE 68 bus NETS-NYPS test system.

\section{FRAMEWORK FOR THE STATISTICAL ASSESSMENT}

The methodology used in this paper is illustrated in Fig. 1, and is based on that proposed in [9]. In this study, only the number of instabilities and the time to instability of generators will be used as the input data for the statistical analysis. Authors in [9] instead analyzed, in a probabilistic manner, transient stability indices based on angle measurements and information obtained from clustering methods [15] for the purpose of identification of critical generators.

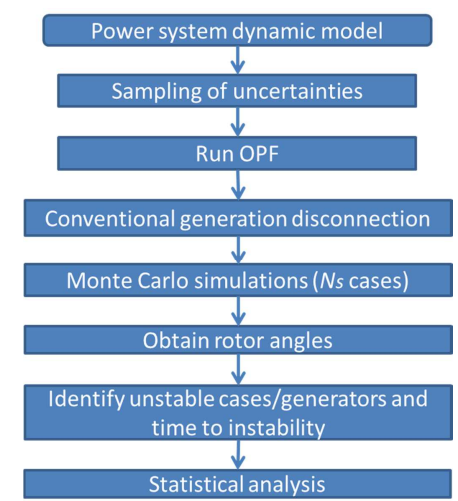

Figure 1. Proposed methodology (adapted from [9])

This work was supported by the EPSRC project HubNet: Research Leadership and Networking for Energy Networks - Extension (EP/N030028/1) and the School of Electrical and Electronic Engineering, The University of Manchester. 


\section{A. Power System Dynamic Model - Test network}

The studied system shown in Fig. 2 is the modified IEEE 68 bus test network with the inclusion of RES generation [9], 16 machine reduced order equivalent model of the New England Test System (NETS, G1-G9), New York Power System (NYPS, G10-G13) and three external systems (G14G16). All generators are represented by full sixth order models and have slow DC excitation (IEEE-DC1A) except for G9 which is equipped with a fast static exciter (IEEE-ST1A) and a power system stabilizer (PSS); the system loads are modelled as constant impedances.

With respect to the RES part of the network, two types are connected to each bus shown in Fig. 2: Doubly Fed Induction Generators (DFIGs), representing wind generators and Full Converter Connected (FCC) units, representing wind generators and PV units, both are treated as aggregate units consisting of individual units of $2 \mathrm{MW}$, with the total number of connected units defined based on the output of the aggregate unit. All the RES modelled have Fault Ride Through (FRT) capabilities and remain connected during a fault. For the present study, $66.67 \%$ of the total RES installed capacity per bus is assumed to be DFIG wind generators, while the remaining $33.33 \%$ are FCCs - which are further considered to be $30 \%$ wind and $70 \%$ PV units. Further details of the system and models used can be found in [9].

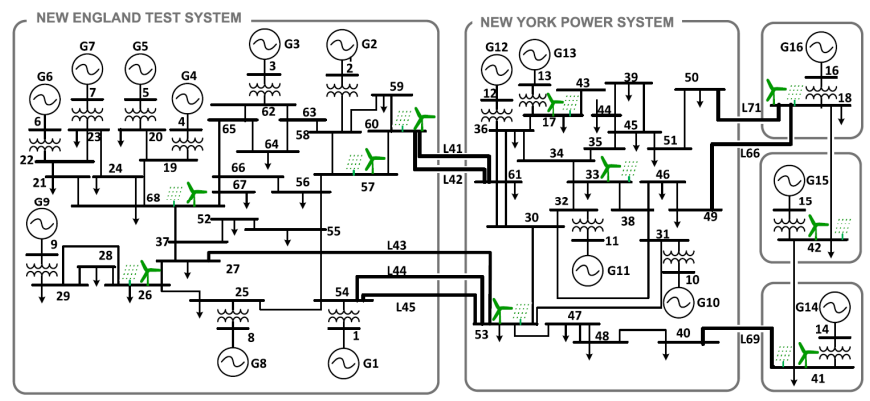

Figure 2. Modified IEEE 68 bus test network with the inclusion of RES

\section{B. Sampling of Uncertanties}

Uncertainties related to system loading and PV generation for a 24 hour period are considered. The hour of the day is sampled randomly using a uniform distribution. In addition, uncertainties for every hour are considered by using a normal distribution for the system loads with a mean value of 1 p.u. and standard deviation of $3.33 \%$ [16], and a beta distribution for the PV generation with $a$ and $b$ parameters equal to 13.7 and 1.3 , respectively $[17,18]$. For the wind generation, the mean value of the wind speed within one day is considered constant [19]. The uncertainty of the wind speed is modelled using a Weibull distribution with parameters $\varphi=11.1$ and $k=$ 2.2 [20]. The power curve of a typical wind generator is used to derive the power output [21].

With respect to fault sampling, only three phase selfclearing faults are considered with a uniform distribution to model the fault location. This approach makes the fault probability at any point along a line, and the probability of a fault in each line of the network, equal. A normal distribution with a mean value of 13 cycles and standard deviation $6.67 \%$ is used to model the fault duration. The fault duration time is selected to generate an appropriate number of stable/unstable cases for the analysis [15]. Further information about the sampling of uncertainties can be found in [9].

\section{Conventional Generation Disconnection}

Optimal Power Flow (OPF) is used to calculate the output of the conventional generators in the system (cost functions adopted from [16]). The inclusion of RES causes a reduction in conventional generation production. The amount of conventional generation disconnection depends on the spare capacity $S C_{i g}$ defined for the system, as specified in (1).

$$
S C_{i g}=1-\frac{P_{S G, i g}}{S_{S G, i g} \times p f_{S G, i g}}
$$

where $P_{S G, i g}$ is the real power of each generator determined by OPF (MW), $S_{S G, i g}$ is the rated apparent power of each generator (MVA) and $p f_{S G, i g}$ is the nominal power factor [9].

For the present study, a fixed $15 \%$ of spare capacity is considered. The displacement of conventional generation capacity and inertia changes due to the production of RES are effectively taken into account by the resulting $S_{S G, i g}$ in (1), since inertia parameters are calculated in p.u. using the rated MVA capacity of the machine. In case the resulting $S_{S G, i g}$ is larger than the initial nominal apparent power of the generators, it is set to the initial nominal value and no conventional generation is disconnected in that case.

\section{Probabilistic Monte Carlo Simulations}

A number of MC simulations, $N_{\mathrm{S}}$, are performed for each scenario after considering all uncertainties. Even though the required number of simulations $\left(N_{\mathrm{S}}\right)$ defined in [9] to achieve the required accuracy was 6000 , in this study the number of simulations $N_{\mathrm{S}}$ is set to 10000 in order to obtain more unstable cases for credible statistical analysis.

Each scenario is defined by the amount of installed capacity of RES given as a percentage of the total installed conventional generation capacity of the system. It is important to highlight that the actual amount of power produced by RES generation will depend on the resulting OPF considering system uncertainties and not on the nominal penetration level. A total of 11 scenarios are generated, from $0 \%$ to $90 \%$ RES penetration in $10 \%$ steps, plus an additional $55 \%$ penetration level. Analysis of penetration levels above $90 \%$ will result in cases in which the RES production levels are enough to provide the entire load of the system, producing an operating condition without the need of conventional generation, which is not the focus of the study in the present paper. Each MC simulation involves a Time Domain Simulation (TDS) of the system subjected to the corresponding three phase fault. The simulation time is set to be $5 \mathrm{~s}$ with the fault applied at $1 \mathrm{~s}$, since the analysis focuses on first swing instability.

\section{Statistical ANALYSIS}

Only two sets of results will be statistically analyzed to study the dynamic behavior of the system, the total number of instabilities and the time to instability. For each scenario, the statistical analysis of the time to instability will be performed by processing the information obtained from MC simulations 


\section{ACCEPTED VERSION OF THE PAPER}

in the form of Probability Density Functions (PDFs), and the calculation of other typical statistical parameters such as the mean of resulting data. The procedure is repeated for each scenario to analyze the impact of RES on transient stability.

\section{A. Total Number of Instabilities per Scenario}

The total number of unstable events is obtained for each scenario. This allows for measuring the impact of each penetration level on transient stability. Furthermore, based on the potential hazard to the network, two types of transient instability event could be defined and analyzed independently.

1) Single-machine unstable cases: when the simulated disturbance results in only one machine losing synchronism for the whole simulation period. In this case, such instability might cause the disconnection of one generator only, causing potential frequency problems, depending on the rated power of the machine with respect to the total generation of the system and its topology. The probability of the widespread loss of supply and system collapse could be deemed to be very low.

2) Multi-machine unstable cases: when the simulated disturbance causes the instability of more than one generator during the simulation period. Here the potential consequences are much more dangerous to the system compared to a singlemachine unstable case. Several machines losing synchronism might cause their disconnection from the network, severe frequency problems and further chain disconnections, leading to a substantial loss of supply and even massive blackouts. These types of event, although of low probability of occurrence, represent a high risk to system operation.

\section{B. Time to Instability of the First Generator}

The time to instability of the first generator losing synchronism (per event), is obtained in order to analyze possible changes on such statistical times for different RES penetration levels. This will help to identify, in a broad way, how the RES penetration level increases or reduces the time to instability. Events with lower instability times are more critical to the system because higher rotor acceleration must be counter-balanced and any attempt to mitigate their instability will be more difficult, since less time is available before generator disconnection due to e.g., pole slipping. For this part, only the time to instability of the first generator is analyzed without taking into account which generator it was.

\section{Total Number of Instabilities per Generator}

The total number of times each generator loses synchronism is a direct indicator of how critical it is for the system. The analysis of the variation of such results for different penetration levels will show the impact of RES on the dynamic behavior of the system. Furthermore, for the most critical multi-machine unstable cases, it is important to have the information about generators losing synchronism first since successful stabilization of those may avoid other generators losing synchronism later. This information will also help the deployment of optimal corrective control. Therefore, the number of instabilities per generator, and which generator(s) lose synchronism first, will be also analyzed.

\section{Time to Instability per Generator}

Besides the number of instabilities per generator, the time to instability of each one will be obtained and analyzed. This information is important for two main reasons. First, the generators presenting statistically less time to instability are definitely critical to the system, as explained before; and second, generators presenting a similar statistical time to instability can be grouped together and corrective measures could be strategically implemented to stabilize the group instead of individual machines, possibly optimizing the deployment of corrective control. This information is more crucial for the stabilization of the most dangerous multimachine unstable events, since in those cases two or more machines lose synchronism in the same event, with a potential further impact of any corrective control applied.

\section{E. Criterion for the Identification of Unstable Generators}

The rotor angles of each generator measured with respect to the angle of a predefined reference machine will be obtained from simulations. A generator will be considered unstable when the condition defined by (2) is reached for the first time within the simulation period.

$$
\text { When }\left|\Delta \delta_{i j-M A X}\right|>360^{\circ} \text {, if } \delta_{i}>\delta_{j} \rightarrow G_{i} \text { is unstable }
$$

where $\delta_{i}$ is the rotor angle of generator $i\left(G_{i}\right)$ and $\Delta \delta_{i j-M A X}$ is the maximum angle difference between any two generators for each instant of time during the simulation. The point in time condition (2) is reached will also be stored for analysis as discussed previously in this section.

With respect to the transient stability limit proposed in (2), the Transient Stability Index (TSI) defined in [22] has been used successfully in the past for transient stability assessment, e.g., [3, 9]. It establishes that the system is unstable if the maximum rotor angle difference between any two generators exceeds a value of $360^{\circ}$, hence the same limit is used in (2) for instability identification.

To elaborate on equation (2), it can be said that based on the actual stability physical phenomenon, the generators losing synchronism after a severe disturbance are the ones accelerating with respect to their initial synchronous rotation at nominal speed. The increase of the rotational speed will not be damped if instability is reached, hence it will not return or oscillate around the initial synchronous speed. As a consequence there will be an uncontrollable angle shift above the initial steady state angle. Therefore, an undamped increase of rotational speed (unequivocal sign of first swing instability) will definitely produce an angle shift in the positive direction (never negative) with respect to their initial position.

Based on the previous analysis, when the angular separation of any two rotor angles exceeds the $360^{\circ}$ threshold as criterion in (2) specifies, the larger rotor angle of the two will be the unstable one. It is important to notice that rotor angles $\delta_{i}$ and $\delta_{j}$ can take either positive or negative values during oscillation; e.g., if the chosen reference machine loses synchronism for a simulated event, the angles of all other (stable) generators will have negative values, while the angle of the reference machine will always be zero; physically, this 


\section{ACCEPTED VERSION OF THE PAPER}

really means that the rotor angle of the reference machine is actually the one deviating towards the positive direction with respect to the other group of stable generators and also with respect to its initial position. The criterion in (2) allows the identification of this fact for any condition and reference machine chosen, hence ensuring the correct identification of machines losing synchronism in the system. The reference generator chosen for the system analyzed in this study is G13.

\section{RESULTS AND DISCUSSION}

\section{A. Total Number of Instabilities per Scenario}

Fig. 3 shows the number of instabilities out of the 10000 MC simulations for different RES penetration levels from $0 \%$ to $90 \%$. The curve indicates that, initially, the introduction of RES reduces the number of instabilities. This positive impact is maintained to about $55 \%$ penetration level, after which the number of instabilities start to increase. The U-shape of the total number of instabilities curve comes completely from multi-machine unstable cases. The number of single-machine unstable cases remains approximately constant (between 7 $8 \%$ ), at least up to a $60 \%$ penetration level, above which they start to increase in an approximate linear fashion.

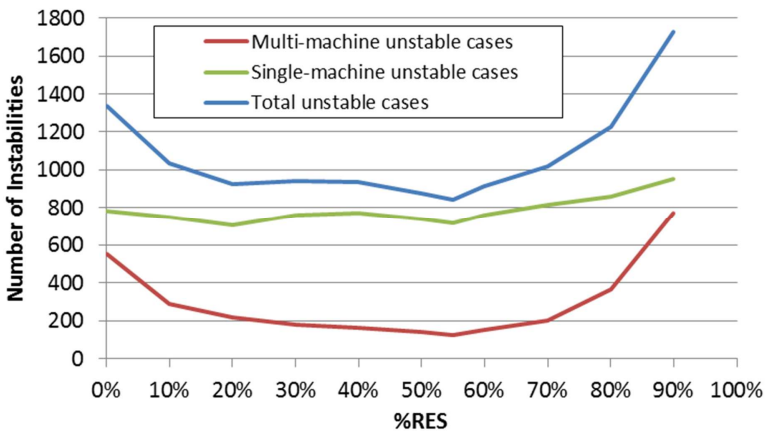

Figure 3. Number of unstable cases for different RES penetration levels

\section{B. Analysis of the First Unstable Generator}

Fig. 4 shows the PDFs of the time to instability of the first generator to lose synchronism per event, for all penetration levels. In general, for higher penetration levels, the peak of the PDFs tends to shift to the left (faster times to instability). The peak of the PDF for 0\% RES is located at 800 ms approx., while the peak of the PDFs for higher penetration levels 70$90 \%$ RES is located at 700-720 ms.

Fig. 5 and Fig. 6 show the time to instability for the singlemachine unstable cases and for the first generator losing synchronism for multi-machine unstable cases, respectively. It is observed that for the single-machine unstable cases, the penetration level has a minor influence in the time to instability, since all the PDFs are similar. With respect to the PDF of the multi-machine unstable cases, for penetration levels of $0 \%$ and $60-90 \%$, there are two distinguishable peaks (present but less prominent for other RES levels) whose influence can also be noticed in the PDF of Fig. 4. Focusing on high $60-90 \%$ RES levels, the second peaks occur at times greater than $2000 \mathrm{~ms}$. Therefore, for penetration levels above $55 \%$, although the number of instabilities starts to increase, the probability of having times to instability above $2 \mathrm{~s}$ for the multi-machine cases also tends to increase. This would give more time for any corrective actions to take place. In addition, for penetration levels between $10 \%$ and $55 \%$, the number of multi-machine instabilities reduces but the probability of having a faster instability time increases. It is concluded that high penetration levels can increase the probability of instability occurrence to later times.

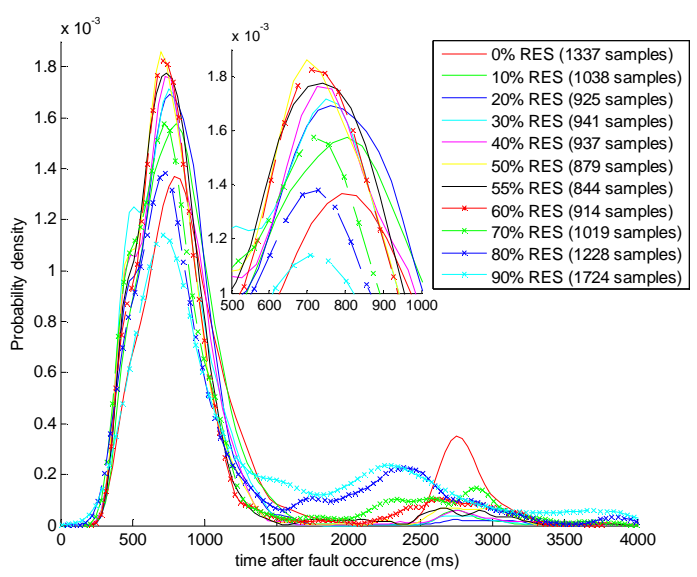

Figure 4. PDFs time to instability first generator, all cases

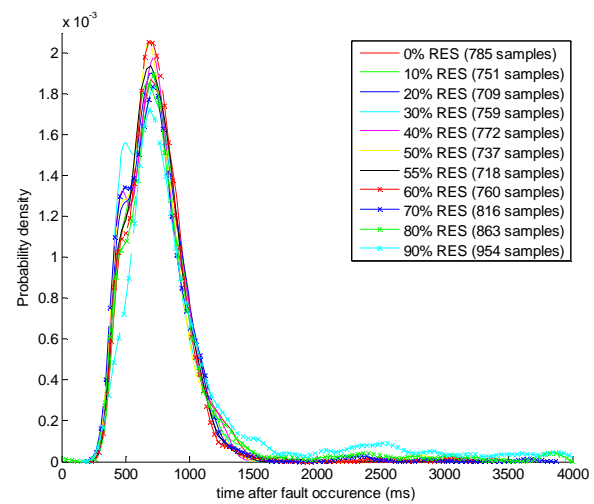

Figure 5. PDFs time to instability, single-machine unstable cases

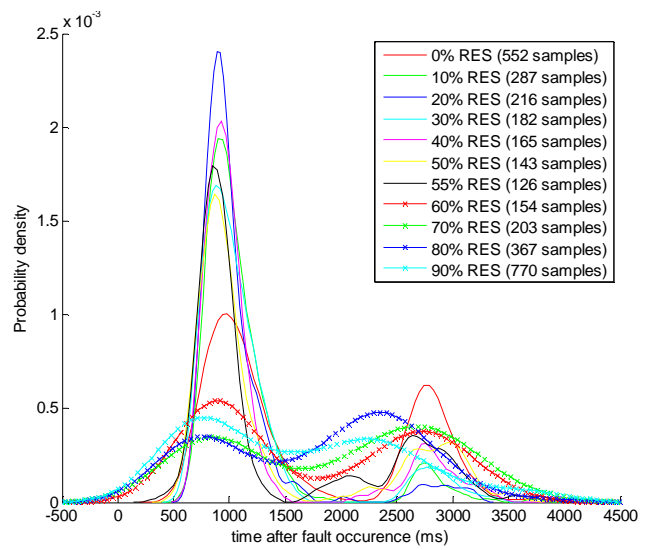

Figure 6. PDFs time to instability, first gen. multi-machine unstable cases

Fig 7 shows the number of times each generator loses synchronism per event only for the single-machine unstable cases. G9 and G11 are the most critical for all penetration levels, while G10 exhibits a very significant increase in the number of instabilities observed for penetration levels of $70 \%$ and above. Fig. 8 shows the number of times each generator is the first one to lose synchronism per event for the multi- 
machine unstable cases. G4 and G5 are the most critical with a higher number of first instabilities for low penetration levels, although decreasing as the penetration levels increase, becoming less important for those scenarios (above 50\% RES). G10 becomes critical for penetration levels of $80 \%$ and above; the same happens with G11 to a lesser extent, while the number of times G16 is the first one to become unstable increases significantly for penetration levels of $60 \%$ and above, becoming more critical than all other generators for these penetration levels. It is observed that in general, higher penetration levels may change the criticality of the generators in the system and even new critical generators appear.

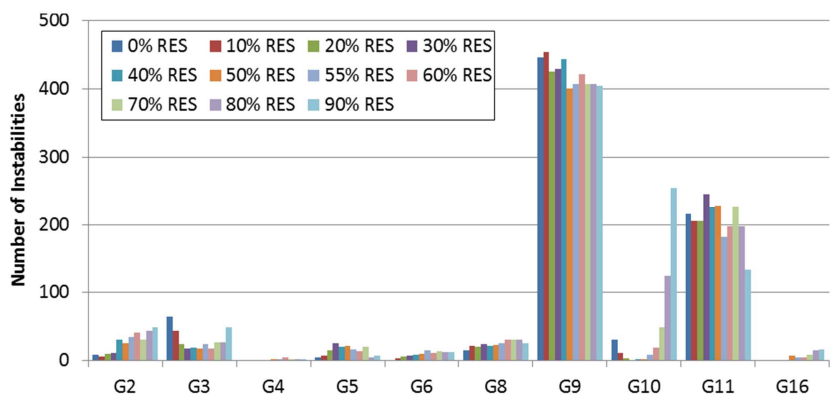

Figure 7. Instabilities per generator, single-machine unstable cases

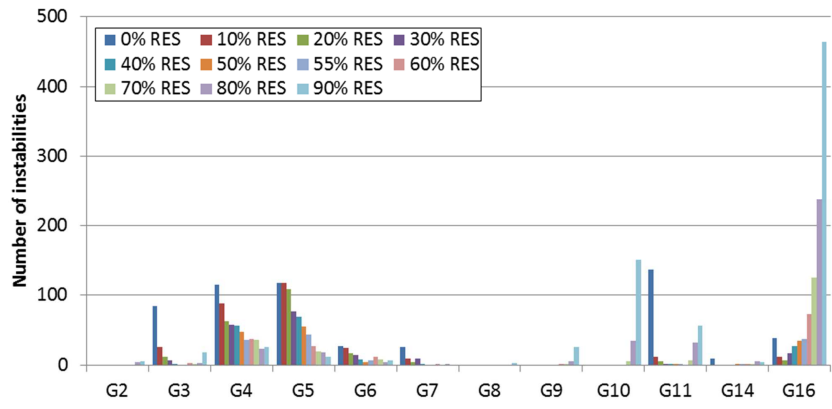

Figure 8. Instabilities per generator, first gen. multi-machine unstable cases

\section{Total Number of Instabilities per Generator}

In this Section the results considering the instability of each generator, whether it is the first or not to exhibit instability, are presented. Fig. 9 shows the total number of instabilities of each generator for all penetration levels, while Fig. 10 shows the number of instabilities of each generator only for the multi-machine unstable cases. Besides the increase in the number of observed instabilities of G10/G11/G16 for higher penetration levels, it is observed that the number of instabilities of G9 also increases considerably with the \%RES, mainly due to its participation in the multimachine unstable cases, not as a first unstable generator (Fig. 10). Also, G14/G15 increase their number of instabilities at the same rate as G16 and they are the most unstable ones as the \%RES approaches the $90 \%$ penetration level. Critical generators G4/G5 reduce their total number of instabilities as the \%RES increases. It is also observed that G6/G7 have a similar number of instabilities compared to G4/G5, especially for lower penetration levels.

Generators with similar times to instability can be grouped together and their statistical parameters analyzed for better assessment. Fig. 11 shows the mean time to instability for five groups of generators that show similar behavior in the multimachine unstable cases. In general, G4/G5 and G6/G7 means tend to decrease as the \%RES increases, and both groups show similar behavior. These four generators in general lose synchronism faster than the others, hence they are always critical for the multi-machine unstable cases in the analyzed system.

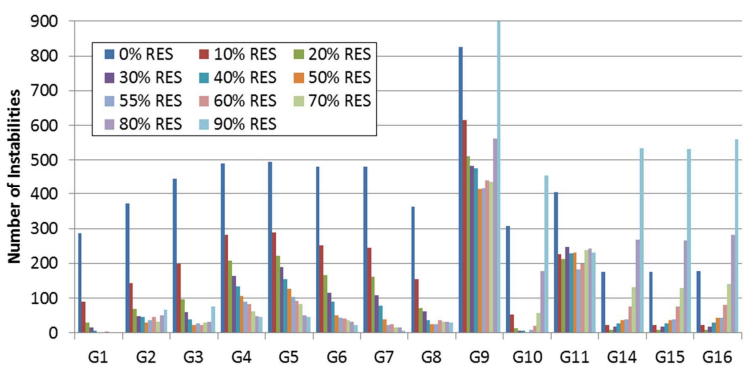

Figure 9. Total number of instabilities per generator, all cases

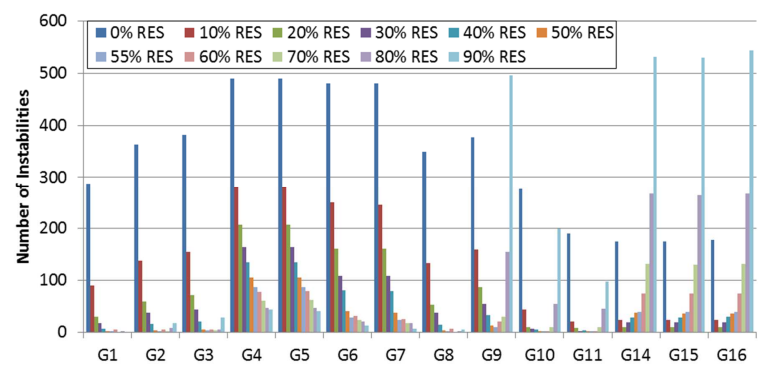

Figure 10. Instabilities per generator, multi-machine unstable cases

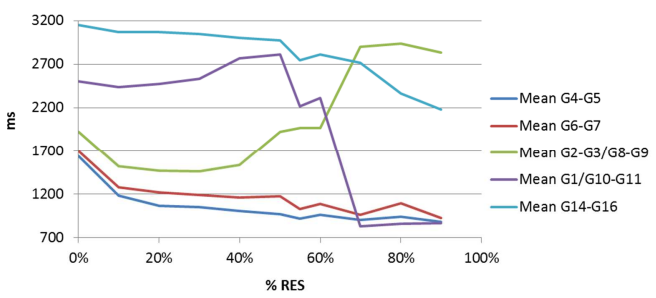

Figure 11. Mean for the times to instability, all cases

In the same way, the group of G2-G3/G8-G9 tends to lose synchronism after G4-G7. For penetration levels above 55\%, the mean time to loss of synchronism of these generators increases considerably, denoting they have become less critical for these penetration levels. The group of G1/G10/G11 tends to lose synchronism above $2000 \mathrm{~ms}$ up to $60 \%$ penetration level, above which the group becomes critical (mostly because of the behavior of G10 and G11 already analyzed), with lower mean values of 600-700 ms. The median and mode of the times to instability for the grouped generators show the same tendency observed for the mean values shown in Fig. 11.

G14-G16 tend to lose synchronism around $3000 \mathrm{~ms}$ for lower penetration levels; as the penetration level increases these time values start to decrease. The importance of this group varies with the scenario. For 0\%RES, there are a considerable number of cases where these generators lose synchronism, although they are not critical as the level of participation in the first unstable cases of G16 is relatively low 
(Fig. 8). This means that this group loses synchronism mostly after others in the multi-machine unstable cases. As the \%RES increases up to $20 \%$, the instabilities of G14-G16 decrease, as well as the participation of G16 as a first unstable generator. For penetration levels of $60-90 \%$, G14-G16 become critical, with an increasing number of total instabilities (Fig. 10) and with G16 being always the first unstable generator for the multi-machine unstable cases (Fig.8). Therefore, the second peak observed in the PDFs for the first generators going unstable above $2000 \mathrm{~ms}$ for high penetration levels (Fig. 6), is caused by the instability of G16, but it can also be said that in general, all three external systems represented by G14-G16 become critical for those scenarios and lose synchronism at approx. the same time without other generators becoming unstable. Hence, the influence of RES is still positive for higher penetration levels in the analyzed system, since the number of instabilities of other generators (in the NETS and NYPS) with lower times to instability are still reduced, those being the ones critical to stabilize due to their low instability times. This adds to the conclusion in Section IV-B about the effect of RES penetration levels, with respect to the increase in the probability of having instability at later times.

\section{CONCLUSIONS}

Probabilistic transient stability assessment was performed for a large network and for a wide range of RES penetration levels, by calculating additional information regarding the time each specific generator exhibits instability in conjunction with the total number of instabilities of the system and per generator. Since instability occurring at faster times is an indication of a more dynamically vulnerable network, the inclusion of the time variable in the analysis allows a more accurate identification of the actual impact of RES on stability, and also of the generators losing synchronism first, which, if acted upon by the implementation of corrective control, may have a substantial impact on transient stability improvement.

Results further show that up to a certain penetration level, the inclusion of RES in the analyzed system is positive because the total number of unstable events is reduced, and that the behavior in the total number of instabilities is more influenced by the more dangerous multi-machine unstable cases, rather than by the single-machine unstable cases. Also, despite an expected increase of unstable events for higher RES levels, it is shown that a positive effect can still be achieved, since the additional unstable cases tend to have times to instability above $2 \mathrm{~s}$, allowing time for the actuation of any corrective control. It was also observed that the statistical times to instability for some generators are similar and could indicate similar transient behavior, which becomes important if corrective control needs to be optimized, since more than one generator could be stabilized by a single measure. It was also shown that for higher penetration levels, new critical generators appear in the system, as others reduce their critical level, indicating that the inclusion of RES in a system changes the pattern of its dynamic behavior.

\section{REFERENCES}

[1] S. Eftekharnejad, V. Vittal, G. T. Heydt, B. Keel, and J. Loehr, "Impact of increased penetration of photovoltaic generation on power systems,"
IEEE Trans.actions on Power Systems, vol. 28, no. 2, pp. 893-901, 2013.

[2] P. G. Bueno, J. C. Hernández, and F. J. Ruiz-Rodriguez, "Stability assessment for transmission systems with large utility-scale photovoltaic units," IET Renewable Power Generation, vol. 10, no. 5, pp. 584-597, 2016.

[3] D. Gautam, V. Vittal, and T. Harbour, "Impact of Increased Penetration of DFIG-Based Wind Turbine Generators on Transient and Small Signal Stability of Power Systems," IEEE Transactions on Power Systems, vol. 24, no. 3, pp. 1426-1434, 2009.

[4] E. Vittal, M. O. Malley, and A. Keane, "Rotor Angle Stability With High Penetrations of Wind Generation," IEEE Transactions on Power Systems, vol. 27, no. 1, pp. 353-362, 2012.

[5] M. Yagami, Y. Ichinohe, Y. Kojima, K. Misawa, and J. Tamura, "Transient stability analysis of synchronous generator in power system with renewable power sources installed," in 2016 19th International Conf. on Electrical Machines and Systems (ICEMS), 2016, pp. 1-5.

[6] J. V. Milanovic, "Probabilistic stability analysis: the way forward for stability analysis of sustainable power systems," Philos Trans A Math Phys Eng Sci, vol. 375, no. 2100, Aug 132017.

[7] S. O. Faried, R. Billinton, and S. Aboreshaid, "Probabilistic evaluation of transient stability of a power system incorporating wind farms," IET Renewable Power Generation, vol. 4, no. 4, pp. 299-307, 2010.

[8] H. Ahmadi and H. Ghasemi, "Maximum penetration level of wind generation considering power system security limits," IET Generation, Transmission \& Distribution, vol. 6, no. 11, pp. 1164-1170, 2012.

[9] P. N. Papadopoulos and J. V. Milanovic, "Probabilistic Framework for Transient Stability Assessment of Power Systems With High Penetration of Renewable Generation," IEEE Transactions on Power Systems, vol. 32, no. 4, pp. 3078-3088, 2017.

[10] S. Xia, X. Luo, K. W. Chan, M. Zhou, and G. Li, "Probabilistic Transient Stability Constrained Optimal Power Flow for Power Systems With Multiple Correlated Uncertain Wind Generations," IEEE Trans. on Sustainable Energy, vol. 7, no. 3, pp. 1133-1144, 2016.

[11] K. Hua, A. Vahidnia, Y. Mishra, and G. Ledwich, "Efficient probabilistic contingency analysis through a stability measure considering wind perturbation," IET Generation, Transmission \& Distribution, vol. 10, no. 4, pp. 897-905, 2016.

[12] P. Ju, H. Li, C. Gan, Y. Liu, Y. Yu, and Y. Liu, "Analytical Assessment for Transient Stability Under Stochastic Continuous Disturbances," IEEE Transactions on Power Systems, vol. PP, no. 99, pp. 1-1, 2017.

[13] J. D. Morales, P. N. Papadopoulos, and J. V. Milanović, "Feasibility of different corrective control options for the improvement of transient stability," in 2017 IEEE Manchester PowerTech, 2017, pp. 1-6.

[14] DIgSILENT PowerFactory 15 User Manual. DIgSILENT GmbH, 2015.

[15] T. Guo and J. V. Milanovic, "Online Identification of Power System Dynamic Signature Using PMU Measurements and Data Mining," IEEE Trans. on Power Systems, vol. 31, no. 3, pp. 1760-1768, 2016.

[16] T. Guo and J. V. Milanović, "Probabilistic Framework for Assessing the Accuracy of Data Mining Tool for Online Prediction of Transient Stability," IEEE Transactions on Power Systems, vol. 29, no. 1, pp. 377-385, 2014.

[17] M. Fan, V. Vittal, G. T. Heydt, and R. Ayyanar, "Probabilistic Power Flow Studies for Transmission Systems With Photovoltaic Generation Using Cumulants," IEEE Transactions on Power Systems, vol. 27, no. 4, pp. 2251-2261, 2012.

[18] S. Tao, Y. Ruoying, Z. Lingzhi, and G. Shan, "Power system probabilistic production simulation containing large-scale wind power and photovoltaic power," in 2013 IEEE PES Asia-Pacific Power and Energy Engineering Conference (APPEEC), 2013, pp. 1-6.

[19] J. P. Coelingh, A. J. M. v. Wijk, and A. A. M. Holtslag, "Analysis of wind speed observations over the North Sea," J. Wind Eng. Ind. Aerodyn., vol. 61, no. 1, pp. 51 - 69, 1996.

[20] R. Preece and J. V. Milanović, "Tuning of a Damping Controller for Multiterminal VSC-HVDC Grids Using the Probabilistic Collocation Method," IEEE Transactions on Power Delivery, vol. 29, no. 1, pp. 318-326, 2014.

[21] Vestas Americas Inc., "V80-2.0 MW: Unsurpassed reliability and performance at high-wind sites in North America," 2011 [Online]. Available: http://www.vestas.com

[22] DSA TOOLS TSAT User Manual. Powertech Labs Inc., Surrey, BC, Canada, 2011. 